\title{
Radiology in the research environment
}

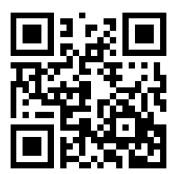

Clinicians and epidemiologists at McMaster University in Canada coined the term 'evidence-based medicine' in 1988. ${ }^{[1]}$ The Scottish physician Dr Archibald Leman Cochrane (1909 - 1988) was a pioneer of evidence-based medicine; the Cochrane Library database of systemic reviews was named after him. Knowledge exchange occurs between researchers and the users of research, whereby research is used to change policy and planning or practice and systems. The translation of best evidence integrated with healthcare practices conduces to saving money and lives.

Translational research is viewed as a solution to the disparity between evidence-based medicine ${ }^{[2,3]}$ in the growing field of basic biomedical research and its application in creating sustainable health solutions ('from bench to bedside'). More recently, it has been viewed as a twoway exchange whereby clinical data and human trials inspire biomedical insights ('from bedside to bench'). ${ }^{[4]}$ There are now several journals dedicated to translational research, including The American Journal of Translational Research and Translational Research.

In imaging research, the process would include the development of more sensitive and specific imaging techniques; assessment of their performance in clinical practice; and investigation of their implementation at an epidemiological level in changing the standard of care in imaging. ${ }^{[3]}$ The translation of research in imaging technology to its application in clinical medicine is a perfect illustration of a successful translational pathway that has had a huge impact on diagnostic and interventional medicine. Magnetic resonance and computed tomography imaging, balloon angioplasty and mammography ${ }^{[5]}$ were ranked among physicians as 3 of the top 5 medical innovations in the last 30 years. To facilitate collaborations between stakeholders in clinical and translational research, institutions such as John Hopkins have established dedicated support structures for translational research.

The World Health Report $2013^{[6]}$ argues that health coverage cannot be achieved without the evidence provided by scientific research. The volume of research on health issues in resource-limited settings has increased, but investigators beyond Africa's borders tend to determine the research agenda. ${ }^{[7,8]}$ It follows that the development and implementation of radiology protocols specific to the type of pathology and trauma encountered in South Africa would have a greater impact on local health priorities. Developing skills to deliver health research is necessary for every country's development. Incorporating these skills at the undergraduate and postgraduate training level is therefore essential.

Radiology incorporated into research performed by other specialties can result in radiologists falling behind competing specialties where high-quality research is performed. The lack of research initiated by the radiologist stems from a lack of funding and dedicated research

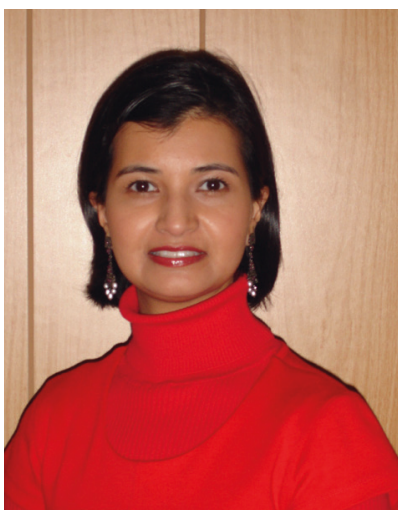

time, as a result of ever-increasing workloads in clinically demanding environments.

In conclusion, then, I wish to emphasise that radiologists assuming a key role in multidisciplinary research communities would be in a position to have a direct and beneficial impact on radiology practices.

\section{Razaan Davis}

Editor

1. Donald A. Evidence-based medicine: Key concepts. Medscape General Medicine 2002;4(2). http://www. medscape.com/viewarticle/430709_3 (accessed 20 October 2013).

2. Lean ME, Mann JI, Hoek JA, et al. Translational research. Br Med J 2008;337:a863. [http://dx.doi. org/10.1136/bmj.a863]

3. Petrou M, Foerster BR, Reich DS. Translational research in radiology: Challenges and roles in a patientbased practice. Acad Radiol 2009;16(5):593-596. [http://dx.doi.org/10.1016/j.acra.2009.01.017]

4. Ledford, H. Translational research, the full cycle. Nature 2008;453:843-845. [http://dx.doi. org/10.1038/453843a]

5. Fuchs VR, Sox HC Jr. Physicians' views of the relative importance of thirty medical innovations. Health Aff (Millwood) 2001;20(5):30-42.

6. World Health Organization. World Health Report 2013: Research for Universal Health Coverage. http:// www.who.int/whr/en/ (accessed 13 October 2013).

7. Gretchen LB, Wiysonge CS, Mills EJ, et al. Global health: the importance of evidence-based medicine. BMC Medicine 2013;11:223. [http://dx.doi.org/10.1186/1741-7015-11-223]

8. Ijsselmuiden C, Marais DL, Becerra-Posada F, Ghannem H. Africa’s neglected area of human resources for health research - the way forward. S Afr Med J 2012;102(4):228-233.

S Afr J Rad 2013;17(4):122. DOI:10.7196/SAJR.1008

\section{CORRECTION}

Please note that the caption to Fig. 3b on page 103 of the August 2013 SAJR is incorrect. It should read:

Fig. 3b. Fetal MRI, coronal T2, in the same fetus, demonstrating enlarged echogenic kidneys. This finding in conjuction with the molar tooth sign is consistent with a diagnosis of the JSRD renal subtype.

The online text has been corrected. The DOI link to the article concerned is: http://dx.doi.org/10.7196/SAJR.899

The publishers of the $S A J R$ apologise for this error. 